PROCEEDINGS OF THE

AMERICAN MATHEMATICAL SOCIETY

Volume 134, Number 5, Pages 1257-1263

S 0002-9939(05)08090-1

Article electronically published on October 4, 2005

\title{
FINITENESS CONDITIONS AND INFINITE MATRIX RINGS
}

\author{
ÁNGEL DEL RÍO AND JUAN JACOBO SIMÓN
}

(Communicated by Martin Lorenz)

\begin{abstract}
For a unital ring $R, \operatorname{RCFM}_{\alpha}(R)$ denotes the ring of row and column finite matrices over $R$ indexed by $\alpha$. We give necessary and sufficient structural conditions on $\operatorname{RCFM}_{\alpha}(R)$ which are equivalent to $R$ being, respectively, Quasi-Frobenius, left artinian, and left noetherian.
\end{abstract}

In this paper $R$ denotes an (associative and unital) ring and $\alpha$ is an infinite set. We use the following notation, where "matrices" means "matrices indexed by $\alpha$ with entries in $R$ ":

$$
\begin{aligned}
A=\mathrm{RFM}_{\alpha}(R) & =\text { ring of row finite matrices, } \\
B=\mathrm{RCFM}_{\alpha}(R) & =\text { ring of row and column finite matrices, } \\
B_{0}=\mathrm{FM}_{\alpha}(R) & =\text { ring of finite matrices. }
\end{aligned}
$$

At first sight it might seem that the rings $A$ and $B$ are too big to reflect properties of the ring $R$, and still more unexpected that $A$ or $B$ could encode finiteness conditions of $R$. However, already in [6, 12] it is shown that the ring $A$ reflects some finiteness conditions of $R$. There is a long tradition in the study of the ring theoretical properties of the $\operatorname{ring} A$ (among others see [2, 6, 9, 12, ). Recently several authors have shown interest in the study of the ring $B$ (see, e.g., 5, 7, 10 ). In this paper we study the properties of the ring $B$ under the assumption that $R$ satisfies some finiteness condition (quasi-Frobenius, artinian, noetherian).

The relationship between a ring $R$ and $A$ comes essentially from the adjoint pair $\operatorname{Hom}_{R}(F,-): R-\bmod \leftrightarrows A-\bmod : F \otimes_{A}-$, where $F$ is a free left $R$-module of rank $|\alpha|$ and $A$ is canonically identified with $\operatorname{End}_{R}(F)$. An interesting exception may be found in the computation of the Jacobson radical (see [13]), where the amount of matrix manipulation exceeds adjunction techniques. If one wants to relate the rings $R$ and $B$ one can also use the adjoint pair $\operatorname{Hom}_{R}(F,-): R-$ $\bmod \leftrightarrows B-\bmod : F \otimes_{B}-$. The difference in the performance of the adjoint pairs for $A$ and $B$ relies on the fact that while $A$ is in the image of $\operatorname{Hom}_{R}(F,-)$, namely $A \simeq \operatorname{End}_{R}(F)$, this is not the case for $B$. However, we may still use some "adjoint-like" techniques to relate some special objects in the category of $R$-modules and some special matrices or ideals of $B$ and keeping in mind that $B$ is the ring of continuous endomorphisms of $F$ in a certain topology [11].

We start with some notation. If $i, j \in \alpha$, then $e_{i j}$ denotes the element of $B_{0}$ having 1 in the $(i, j)$-th entry and zeroes elsewhere, and if $a \in A$, then $a(i, j)$ denotes the $(i, j)$-th entry of $a$. Set $e_{i}=e_{i i}=e_{\{i\}}$. If $F$ is a subset of $\alpha$, then

Received by the editors September 2, 2004 and, in revised form, November 19, 2004

2000 Mathematics Subject Classification. Primary 16S50; Secondary 16P20, 16P40.

Both authors were partially supported by the D.G.I. of Spain and Fundación Séneca of Murcia.

(c)2005 American Mathematical Society 1257

Reverts to public 
set $e_{F}=\sum_{i \in F} e_{i}$. A careful study of the arithmetic of the rings $A, B$ and $B_{0}$ and their idempotents $e_{F}$ for $F$ finite leads to the following lemma.

Lemma 1. $\quad 1 . B_{0}$ is a two-sided ideal of $B$ and a right ideal of $A$.

2. For every $x \in B_{0}$ there is $e=e^{2} \in B_{0}$ such that $x=e x=x e$.

3. The map $\rho: A \rightarrow \operatorname{End}\left({ }_{B_{0}} B_{0}\right)$ that associates $a \in A$ with the endomorphism $\rho_{a}$ of $B_{0}$ given by $\rho_{a}(x)=x a$ is a ring isomorphism.

We need the following module theoretical lemma (see [4, Exercise 18.17]).

Lemma 2. A left $R$-module $M$ is quasi-injective if and only if $f(M) \subseteq M$ for every endomorphism $f$ of the injective hull of $M$.

Recall that $R$ is said to be quasi-Frobenius (QF) if $R$ is right and left artinian, and there exists a duality between the categories of finitely generated left and right $R$-modules. Further, $R$ is said to be quasi-continuous if $e(R) \subseteq R$ for every idempotent endomorphism $e$ of the injective hull of ${ }_{R} R$.

In 6] it is shown that $R$ is QF if and only if $A$ is left self-injective. In contrast, the ring $B$ cannot be even left or right quasi-continuous 7 for any ring $R$. Our first result shows how to compute the injective hull of ${ }_{B} B$ if $R$ is QF. Note that as a consequence of the next theorem any of the equivalent conditions 2-6 hold for some infinite set $\alpha$ if and only if they hold for every infinite set.

Theorem 3. The following conditions are equivalent for a ring $R$ and an infinite set $\alpha$ :

1. $R$ is quasi-Frobenius.

2. $\operatorname{RFM}_{\alpha}(R)$ is left self-injective.

3. $\mathrm{FM}_{\alpha}(R)$ is an injective object in the category $\mathrm{FM}_{\alpha}(R)-\bmod$ consisting of those $\mathrm{FM}_{\alpha}(R)$-modules $M$ such that $\mathrm{FM}_{\alpha}(R) M=M$.

4. $\mathrm{FM}_{\alpha}(R)$ is quasi-injective as a left $\mathrm{RCFM}_{\alpha}(R)$-module.

5. $\operatorname{RFM}_{\alpha}(R)$ is injective as a left $\operatorname{RCFM}_{\alpha}(R)$-module.

6. $\operatorname{RFM}_{\alpha}(R)$ is the injective hull of the regular left $\operatorname{RCFM}_{\alpha}(R)$-module $\operatorname{RCFM}_{\alpha}(R)$.

Proof. Let $A, B$ and $B_{0}$ be as in (1) and let $E$ denote the injective hull of ${ }_{B} B$.

The equivalence between conditions 1 and 2 was proved in [6].

The equivalence between conditions 5 and 6 follows from the fact that $B_{0}$ is essential in ${ }_{B} A$ and hence $E=E\left({ }_{B} A\right)=E\left({ }_{B} B_{0}\right)$.

The fact that condition 3 implies 4 follows from the equality $B_{0}=B B_{0}$.

Identifying $A$ with $\operatorname{End}_{R}\left(R^{(\alpha)}\right)$ one can consider $R^{(\alpha)}$ as a $R-B_{0}$-bimodule and it is well known that the functor $F=B_{0} \operatorname{Hom}_{R}\left(R^{(\alpha)},-\right): R-\bmod \rightarrow B_{0}-$ mod is an equivalence of categories such that $F\left(R^{(\alpha)}\right)=B_{0}$. (Alternatively one can prove this by using the results of [1] or 3.) Now the equivalence between conditions 1 and 3 follows from the fact that $R$ is quasi-Frobenius if and only if ${ }_{R} R^{(\alpha)}$ is injective; this is a direct consequence of Theorems 24.18 and 24.20 of $[8$.

Now we prove that condition 4 implies 6 . Assume that ${ }_{B} B_{0}$ is quasi-injective. We have already seen that $A \subseteq E$. Let $e \in E$ and consider the map $f: B_{0} \rightarrow E$ given by $f(x)=x e$. By assumption and Lemmas 1 and 2 there is an $a \in A$ such that $x e=x a$ for every $x \in B_{0}$. We claim that $e=a$ and this completes the proof. Indeed, if $e \neq a$, then $B(e-a) \neq 0$ and since $B_{0}$ is essential in ${ }_{B} E$ there is $0 \neq x \in B_{0} \cap B(e-a)$. Thus there is $x_{0} \in B_{0}$ and $b \in B$ such that $x=x_{0} x=b(e-a)$ and hence $x=x_{0} x=x_{0} b(e-a)=0$, a contradiction. 
Finally we prove that condition 5 implies 3 . Assume that ${ }_{B} A$ is injective and let $f: N \rightarrow B_{0}$ be a homomorphism of $B_{0}$-modules with $N$ a submodule of $B_{0}$. By using $B_{0} N=N$ and $B_{0}=B_{0} B=B B_{0}$ one deduces that $f$ is a homomorphism of $B$-modules and hence $f$ extends to an endomorphism $g$ of ${ }_{B} A$. If $x \in B_{0}$, then there is $e \in B_{0}$ such that $x=e x$ and hence $g(x)=e g(x) \in B_{0}$. Therefore $g$ restricts to an endomorphism of ${ }_{B} B_{0}$ which extends $f$. This shows that ${ }_{B} B_{0}$ is quasi-injective by Lemma 2 .

For every $i \in \alpha$, consider the map $\pi_{i}: B \rightarrow R^{(\alpha)}$ that associates to $a \in B$, the $i$-th row of $a$. Let $I$ be a left ideal of $B$. Since $\pi_{j}(a)=\pi_{i}\left(e_{i j} \cdot a\right), \pi_{i}(I)$ does not depend on $i$. Write $\pi(I)=\pi_{i}(I)$ for $i \in \alpha$ arbitrary. Clearly $\pi(I)$ is a submodule of $R_{R} R^{(\alpha)}$. (There is an alternative definition of $\pi(I)$ for $I$ a left ideal of $B$ as $R^{(\alpha)} I$, where $R^{(\alpha)}$ is considered as an $R$-B-bimodule in the natural matricial way.) Thus if $a \in B$, then $\pi(A a)$ is the submodule of $R^{(\alpha)}$ generated by the rows of $a$. If $M$ is a submodule of ${ }_{R} R^{(\alpha)}$, then $\iota(M)=\{a \in B: \pi(a) \in M$, for every $i \in \alpha\}$ is a left ideal of $B$.

Definition 4. We say that a left ideal $I$ of $B$ is closed if for every $a \in B \backslash I$ there is $i \in I$ such that $\pi_{i}(a) \notin \pi(I)$.

Lemma 5. A left ideal $I$ of $B$ is closed if and only if $I=\iota(M)$ for some submodule $M$ of ${ }_{R} R^{(\alpha)}$. In particular the left annihilators $l_{B}(X)$ in $B$ of subsets $X$ of $B$ and the left ideals of the form $B(J)=\{a \in B: a(i, j) \in J$ for every $i, j\}$, where $J$ is a left ideal of $R$, are closed left ideals of $B$.

Proof. Clearly $I \subseteq \iota(\pi(I))$ and $I$ is closed if and only if the equality holds. Further if $M$ is a submodule of $R^{(\alpha)}$, then $\iota(\pi(\iota(M)))=\iota(M)$. This proves the first statement. The second is a consequence of the first because $B(J)=\iota\left(J^{(\alpha)}\right)$ and if $X \subseteq B$, then $l_{B}(X)=\iota(M)$ where $M$ is the annihilator in $R^{(\alpha)}$ of $X$.

Proposition 6. The following conditions are equivalent for a left ideal $J$ of $R$ :

1. $\operatorname{RCFM}_{\alpha}(J)=\left\{a \in \operatorname{RCFM}_{\alpha}(R): a(i, j) \in J\right.$ for every $\left.i, j\right\}$ is a cyclic left ideal of $\operatorname{RCFM}_{\alpha}(R)$.

2. $\operatorname{RCFM}_{\alpha}(J)$ is a finitely generated left ideal of $\operatorname{RCFM}_{\alpha}(R)$.

3. $J$ is a finitely generated left ideal of $R$.

Proof. Let $A, B$ and $B_{0}$ as in (11). The equivalence between conditions 1 and 2 follows from the fact that $B$ has single basis number, i.e. ${ }_{B} B \simeq{ }_{B} B^{n}$ for every positive integer $n$. If ${ }_{B} B(J)$ is generated by $X$, then $J$ is generated by the entries of the first row of the elements of $X$. This proves that condition 2 implies 3 .

Let $x_{1}, \ldots, x_{n}$ generate ${ }_{R} J$. Since $\alpha$ is infinite there is a natural identification between $B(J), \operatorname{RCFM}_{\alpha}\left(M_{n, 1}(K)\right)$ and $\operatorname{RCFM}_{\alpha}\left(M_{1, n}(R)\right)$ that we are going to use without specific mention. Let $a \in B(J)$ be the following "diagonal" matrix (diagonal in $\left.M_{\alpha}\left(M_{n, 1}(J)\right)\right)$ :

$$
g=\left(\begin{array}{cccc}
X & 0 & 0 & \cdots \\
0 & X & 0 & \cdots \\
0 & 0 & X & \cdots \\
\vdots & \vdots & \vdots & \ddots
\end{array}\right) \quad \text { with } \quad X=\left(\begin{array}{c}
x_{1} \\
x_{2} \\
\vdots \\
x_{n}
\end{array}\right)
$$


If $b \in B(J)$, then for every $x, y \in J$ there is $c(x, y) \in M_{1, n}(R)$ such that the $(x, y)$-th entry $b(x, y)$ of $b$ is $c(x, y) X$ and we may take $c(x, y)=0$ if $b(x, y)=0$. Then $c=(c(x, y))_{x, y \in \alpha} \in B$ and $b=c a$. This proves that $B(J)=B a$.

Remark 7. If RCFM is replaced by RFM in conditions 1 and 2 of Proposition 6 then they are still equivalent, since $\operatorname{RFM}_{\alpha}(R)$ has a single basis number. However they are not equivalent to condition 3 . For example, if $J$ is the ideal generated by a subset $X$ of $R$, then $\operatorname{RFM}_{X}(J)$ is the cyclic left ideal generated by the matrix having the elements of $X$ in one column and zeroes in the remaining columns.

The Jacobson radical of $R$ is denoted by $J(R)$. The ring $R$ is said to be a Baer ring if any left (equivalently, right) annihilator in $R$ of a subset of $R$ is a direct summand.

Theorem 8. If $\alpha$ is an infinite set, then a unital ring $R$ is left artinian if and only if $J\left(\operatorname{RCFM}_{\alpha}(R)\right)$ is cyclic as a left ideal of $\operatorname{RCFM}_{\alpha}(R)$, and

$$
\operatorname{RCFM}_{\alpha}(R) / J\left(\operatorname{RCFM}_{\alpha}(R)\right)
$$

is a Baer ring. In this case $J\left(\operatorname{RCFM}_{\alpha}(R)\right)=\operatorname{RCFM}_{\alpha}(J(R))$.

Proof. Assume that $R$ is left artinian. By Proposition 6, $\operatorname{RCFM}_{\alpha}(J(R))$ is a cyclic left ideal of $\operatorname{RCFM}_{\alpha}(R)$. By [7, Theorem 14], $\operatorname{RCFM}_{\alpha}(R) / J\left(\operatorname{RCFM}_{\alpha}(R)\right)$ is a Baer ring, and $J\left(\operatorname{RCFM}_{\alpha}(R)\right)=\operatorname{RCFM}_{\alpha}(J(R))$, so that $J\left(\operatorname{RCFM}_{\alpha}(R)\right)$ is a cyclic left ideal of $\operatorname{RCFM}_{\alpha}(R)$.

Conversely, by [7, Theorem 14], $R$ is a perfect ring and $J\left(\operatorname{RCFM}_{\alpha}(R)\right)=$ $\operatorname{RCFM}_{\alpha}(J(R))$. By hypothesis and Proposition [6, $J(R)$ is a finitely generated left ideal of $R$. Then by [4, Ex. 28.9] $R$ is left artinian.

By Proposition 6 if every closed left ideal of $B$ is finitely generated, then $R$ is left noetherian. Our next result proves the converse for $\alpha$ countable.

Theorem 9. The following conditions are equivalent for a unital ring $R$ :

1. $R$ is left noetherian.

2. Every closed left ideal of $\operatorname{RCFM}_{\mathbb{N}}(R)$ is finitely generated.

3. Every closed left ideal of $\operatorname{RCFM}_{\mathbb{N}}(R)$ is cyclic.

Proof. By Proposition 6 we only have to prove that condition 1 implies 3.

Let $I=\iota(M)$ be a closed left ideal of $B=\operatorname{RCFM}_{\mathbb{N}}(R)$. For every non-negative integer $n$ let $p_{n}: R^{(\mathbb{N})} \rightarrow R^{(\mathbb{N})}$ be the projection on the first $n$ coordinates and let

$$
M_{n}=M \cap \operatorname{Im} p_{n} \quad \text { and } \quad K_{n}=M \cap \operatorname{ker} p_{n} .
$$

Note that

$$
0=M_{0} \leq M_{1} \leq M_{2} \leq \ldots \leq \bigcup_{n \geq 0} M_{n}=M=K_{0} \geq K_{1} \geq K_{2} \geq \ldots \geq \bigcap_{n \geq 0} K_{n}=0 .
$$

If $M$ is finitely generated, say $M=\left\langle x_{1}, \ldots, x_{k}\right\rangle$, then $I$ is generated by the matrix

$$
a=\left(\begin{array}{c}
x_{1} \\
x_{2} \\
\vdots \\
x_{k} \\
0 \\
\vdots
\end{array}\right) .
$$


(Note that the rows of $a$ are elements of $R^{(\mathbb{N})}$ and hence $a \in B$.) Thus assume that $M$ is not finitely generated. This implies that $M \neq M_{n}$ for every $n \geq 0$.

If $n \geq 0$, then $p_{n}(M)$ is finitely generated and hence $p_{n}(M)=p_{n}\left(\left\langle m_{1}, \ldots, m_{k}\right\rangle\right)$ for some $m_{1}, \ldots, m_{k} \in M$. Since $M$ is not finitely generated there is $m \in M \backslash$ $\left\langle m_{1}, \ldots, m_{k}\right\rangle$. On the other hand $p_{n}(m)=\sum_{i=1}^{k} r_{i} p_{n}\left(m_{i}\right)$ for some $r_{i} \in R$ and thus $0 \neq m-\sum_{i=1}^{k} r_{i} m_{i} \in K_{n}$.

The argument of the previous paragraph shows that $K_{n} \neq 0$ for every $n \geq 0$, and using this fact we recursively construct a sequence $\left(m_{n}, k_{n}, S_{n}\right)_{n \geq 0}$ as follows:

$$
\begin{aligned}
k_{0} & =0, \text { the integer; } \\
S_{0} & =0, \text { the trivial submodule of } M ; \\
m_{n} & =\min \left\{m: M_{m} \cap K_{k_{n}} \neq 0\right\} ; \\
k_{n+1} & =\min \left\{k:\left(M_{m_{n}}+\sum_{i=0}^{n} S_{i}\right) \cap K_{k}=0\right\}
\end{aligned}
$$

and $S_{n+1}$ is a finitely generated submodule of $K_{k_{n}}$ such that

$$
\begin{gathered}
\left(M_{m_{n+1}}+\sum_{i=0}^{n} S_{i}\right) \cap K_{k_{n}} \subseteq S_{n+1} \subseteq K_{k_{n}} \quad \text { and } \\
\left(S_{n+1}+K_{k_{n+1}}\right) / K_{k_{n+1}}=K_{k_{n}} / K_{k_{n+1}} .
\end{gathered}
$$

The existence of $m$ such that $M_{m} \cap K_{k_{n}} \neq 0$ is warranted by the fact that $K_{k_{n}} \neq 0$; the existence of $k$ such that $\left(M_{m_{n}}+\sum_{i=0}^{n} S_{i}\right) \cap K_{k}=0$ follows from the fact the $M_{n}+\sum_{i=0}^{n} S_{i}$ is finitely generated and hence it is embedded in $K_{k}$ for some $k$; finally the existence of $S_{n+1}$ finitely generated and satisfying (2) follows from the fact that $M_{m_{n+1}} \cap K_{k_{n}}$ and $K_{k_{n}} / K_{k_{n+1}}$ are finitely generated. Since $M_{m_{n}} \cap K_{k_{n}} \neq 0$, $M_{m_{n}} \cap K_{k_{n+1}} \neq 0$ and $M_{m_{n+1}} \cap K_{k_{n+1}} \neq 0, m_{n}<m_{n+1}$ and $k_{n}<k_{n+1}$ for every $n$, i.e. $\left(m_{n}\right)$ and $\left(k_{n}\right)$ are increasing sequences.

Claim: For every $n, r \geq 0,\left(M_{m_{n}}+\sum_{i=0}^{n} S_{i}\right) \cap K_{k_{r}} \subseteq \sum_{i \geq r+1}^{n+1} S_{i}$.

In particular, $K_{k_{r}} \subseteq \sum_{i \geq r+1} S_{i}$ for every $r \leq 0$.

If $n-r<0$, then $\left(M_{m_{n}}+\sum_{i=0}^{n} S_{i}\right) \cap K_{k_{r}} \subseteq\left(M_{m_{n}}+\sum_{i=0}^{n} S_{i}\right) \cap K_{k_{n+1}}=0$ and the Claim is obvious, so we assume that $n-r \geq 0$ and argue by induction on $n-r$. If $n-r=0$, then $\left(M_{m_{n}}+\sum_{i=0}^{n} S_{i}\right) \cap K_{k_{r}}=\left(M_{m_{r}}+\sum_{i=0}^{r} S_{i}\right) \cap K_{k_{r}} \subseteq S_{r+1}$ by construction. Assume that $n-r>0$ and let $m \in\left(M_{m_{n}}+\sum_{i=0}^{n} S_{i}\right) \cap K_{k_{r}}$. Then there is $s_{r+1} \in S_{r+1}$ such that $n=m-s_{r+1} \in K_{k_{r+1}}$. Thus $n \in\left(M_{m_{n}}+\sum_{i=0}^{n} S_{i}\right) \cap$ $K_{k_{r+1}}$, by induction hypothesis $n \in \sum_{i \geq r+2}^{n+1} S_{i}$ and so $m \in \sum_{i \geq r+1}^{n+1} S_{i}$. This proves the Claim.

For every $n$ let $X_{n}$ be a finite generating set of $S_{n}$ and construct the matrix

$$
a=\left(\begin{array}{c}
X_{1} \\
X_{2} \\
X_{3} \\
\vdots
\end{array}\right) .
$$

That is, the first rows of $a$ are formed by the elements of $X_{1}$, in some order, the next rows are formed by the elements of $X_{2}$, etc. Since $S_{n} \subseteq K_{k_{n}}$ and $\left(k_{n}\right)$ is a strictly increasing sequence, each column of $a$ has only finitely many non-zero entries. That is, $a \in B$ and because $I$ is a closed left ideal and every row of $a$ belongs to $M=\pi(I)$, one deduces that $a \in I$.

Let $u_{n}$ be the cardinality of $X_{n}$ and let $v_{n}=\sum_{i=1}^{n} u_{n}$. By the claim if $x \in K_{r}$, then $x=y a$ for some $y \in \operatorname{ker} p_{v_{r}}$. If $x \in I$, then there is a strictly increasing 
sequence $\left(r_{n}\right)_{n}$ such that $\pi_{m}(x) \in K_{k_{n}}$ for every $m \geq r_{n}$. Thus if $r_{n} \leq m<r_{n+1}$, then $\pi_{m}(x)=y_{m} a$ with $y_{m} \in \operatorname{ker} p_{v_{r}}$ and hence $x=y a$, where $y$ is the row finite matrix defined by setting $\pi_{m}(y)=y_{m}$. Since $M=\sum_{n>1} S_{n}$ is not finitely generated the sequence $\left(v_{n}\right)$ is non-decreasing and non-bounded and this implies that $y \in B$. Thus $x \in B a$, and this proves that $I=B a$.

Note that the proof of Theorem 9 does not apply if the index set is not countable. We do not know whether or not the closed ideals of $B_{\alpha}(R)$ are cyclic for $R$ noetherian and $\alpha$ a non-countable set. A consequence of Theorem 9 is the following.

Corollary 10. If $R$ is left noetherian, then $\operatorname{RCFM}_{\mathbb{N}}(R)$ is left coherent.

Proof. Let $I$ be a finitely generated left ideal of $B$. Since $B$ has single basis number there is a surjective homomorphism $f: B \rightarrow I$ of left $B$-modules. Then $I=B a$ for some $a \in B$ and hence Ker $f$ is the left annihilator of $a$. Thus Ker $f$ is a closed left ideal of $B$. By Theorem 9, Ker $f$ is finitely generated and we conclude that $I$ is finitely presented.

We conjecture that the converse of Corollary 10 is false in general. However, we provide a partial converse in the next proposition.

Proposition 11. Let $\alpha$ be an infinite set and let $R$ be a unital ring. If $\operatorname{RCFM}_{\alpha}(R)$ is left coherent, then $R$ is left coherent and satisfies acc on direct summands.

Proof. Assume that $B=\operatorname{RCFM}_{\alpha}(R)$ is left coherent. To prove that $R$ is left coherent we show that if $a_{1}, \ldots, a_{r} \in R$, then the kernel of the homomorphism $f: R^{n} \rightarrow R$ given by $f\left(x_{1}, \ldots, x_{n}\right)=\sum_{i=1}^{n} x_{i} a_{i}$ is finitely generated. Let $J=$ $\left\{j_{1}, \ldots, j_{n}\right\}$ be a subset of $\alpha$ of cardinality $n$ and $a \in B$ be given by

$$
a(x, y)= \begin{cases}1, & \text { if } x=y \notin J \\ a_{i}, & \text { if } x=j_{i} \text { and } y=j_{1}, \\ 0, & \text { otherwise. }\end{cases}
$$

By the construction of $a$, the left annihilator $l_{B}(a)$ of $a$ in $B$ is formed by the element $b \in B$ such that $b(x, y)=0$ if $y \notin J$ and $\left(b\left(x, j_{1}\right), \ldots, b\left(x, j_{n}\right)\right) \in \operatorname{ker} f$ for every $x \in \alpha$. By hypothesis there is $b \in B$ such that $B b=l_{B}(a)$ and hence it is easy to see that ker $f$ is generated by the elements of the form $b(x)=\left(b\left(x, j_{1}\right), \ldots, b\left(x, j_{n}\right)\right)$ with $x \in \alpha$. Since $b \in B, b(x)=0$ for almost all $x \in \alpha$ and hence ker $f$ is finitely generated as wanted.

Now we prove that $R$ satisfies acc on direct summands. Otherwise $R$ has an infinite countable set $\left\{f_{1}, f_{2}, \ldots\right\}$ of non-zero orthogonal idempotents. Let $J=$ $\left\{j_{1}, j_{2}, \ldots\right\}$ be an infinite countable subset of $\alpha$ and consider the matrix $a \in B$ given as follows:

$$
a(x, y)= \begin{cases}1, & \text { if } x=y \notin J, \\ 1-f_{n}, & \text { if } x=y=j_{n}, \\ -f_{n+1}, & \text { if } x=j_{n+1} \text { and } y=j_{n}, \\ f_{n+1}-1, & \text { if } x=j_{n+2} \text { and } y=j_{n}, \\ 0, & \text { otherwise. }\end{cases}
$$

By hypothesis $l_{B}(a)=B b$ for some $b \in B$. Let $I=\sum_{n \in \mathbb{N}} R f_{i}$ and let $K$ be the left ideal of $R$ generated by the entries in the $j_{1}$-column of $b$. Note that $K$ is finitely generated while $I$ is not. We are going to obtain a contradiction by showing that $I=K$. 
To prove $I \subseteq K$, consider, for any $n \in \mathbb{N}$, the matrix $m_{n} \in B$ having $f_{n}$ in the entries $\left(j_{1}, j_{i}\right)$ for $1 \leq i \leq n$ and 0 in any other entry. Note that $m_{n} a=0$ and hence $m_{n} \in B b$. Thus $f_{n} \in K$.

The reverse inclusion follows by showing that all the entries of $b$ belong to $I$. (In fact the same holds for any element in $\left.l_{B}(a)\right)$. Fix $x \in \alpha$ and let $Y=\{y \in$ $\alpha: b(x, y) \neq 0\}$. We have to prove that $b(x, y) \in I$ for every $y \in Y$. This is obvious if $Y=\emptyset$, so assume that this is not the case. Having in mind that $b a=0$ one has that $Y \subseteq J$. Let $n$ be the maximum positive integer such that $b\left(x, j_{n}\right) \neq 0$. We prove that $b\left(x, j_{m}\right) \in I$ for $1 \leq m \leq n$ by induction on $m-n$. First $0=(b a)\left(x, j_{n}\right)=b\left(x, j_{n}\right)\left(1-f_{n}\right)$ and hence $b\left(x, j_{n}\right) \in R f_{n} \subseteq I$. Second $0=(b a)\left(x, j_{n-1}\right)=b\left(x, j_{n-1}\right)\left(1-f_{n-1}\right)-b\left(x, j_{n}\right) f_{n}$ and hence $b\left(x, j_{n-1}\right) \in R f_{n-1}+$ $R f_{n} \subseteq I$. Finally, if $1 \leq m \leq n-2$, then $0=(b a)\left(x, j_{m}\right)=b\left(x, j_{m}\right)\left(1-f_{j_{m}}\right)-$ $b\left(x, j_{m+1}\right) f_{m+1}+b\left(x, j_{m+1}\right)\left(f_{m+1}-1\right)$ and hence $b\left(x, j_{m}\right) \in I$, because by the induction hypothesis $b\left(x, j_{m+1}\right) \in I$.

Corollary 12. If $\alpha$ is an infinite cardinal and $R$ is a ring, then $R$ is semisimple artinian if and only if $R$ is von Neumman regular and $\operatorname{RCFM}_{\alpha}(R)$ is left coherent.

Proof. This is a direct consequence of Proposition 11 and the fact that $R$ is semisimple artinian if and only if $R$ is von Neumann regular and satisfies acc on direct summands [8, Theorem 19.26 A].

\section{REFERENCES}

[1] G.D. Abrams, Morita equivalence for rings with local units, Comm. Algebra 11 (1983) 801837. MR.0695890 (85b:16037)

[2] G. Abrams and J. Haefner, Picard groups and infinite matrix rings, Trans. Amer. Math. Soc. 350 (1998) 2737-2752. MR:1422591 (98j:16017)

[3] P.N. Ánh and L. Márki, Morita equivalence for rings without identity, Tsukuba J. Math. 11 (1987) 1-16. MR0899719 (88h:16054)

[4] F.W. Anderson and K.R. Fuller, Rings and categories of modules, Springer-Verlag, 1974. MR0417223 (54:5281)

[5] P. Ara, E. Pardo and F. Perera, The structure of countably generated projective modules over regular rings, J. Algebra 226 (2000) 161-190. MR.1749882 (2001a:16017)

[6] G.M. Brodskii, Endomorphism rings of free modules, Mat. Sb. 94(136) (1974) 226-242. Math. USSR Sbornik 23(2) (1974). MR.0349761 (50:2254)

[7] V. Camillo, F.J. Costa-Cano and J.J. Simón, Relating properties of a ring and its ring of row and column finite matrices, J. Algebra 224 (2001) 435-449. MR1859035 (2002i:16039)

[8] C. Faith, Algebra II. Ring Theory, Springer-Verlag, 1976. MR0427349 (55:383)

[9] P. Menal, On the endomorphism ring of a free module, Publ. Sec. Mat. Univ. Autònoma Barcelona 27 (1983), no. 1, 141-154. MR0763863 (86g:16046)

[10] K.C. O'Meara, The exchange property for row and column-finite matrix rings, J. Algebra 268 (2003) no. 2, 744-749. MR2009331 (2004i:16040)

[11] D. Ornstein, Dual vector spaces, Ann. Math. 69 (1959) 520-534. MR0107153 (21:5878)

[12] B. L. Osofsky, Some properties of rings reflected in endomorphism rings of free modules, Cont. Math. 13 (1982), 179-181. MR.0685934 (84a:00007)

[13] R. Ware and J. Zelmanowitz, The Jacobson radical of the endomorphism ring of a projective module, Proc. AMS 26 (1970) 15-20. MR0262281(41:6891)

Departamento de Matemáticas, Universidad de Murcia, 30100 Murcia, Spain

E-mail address: adelrio@um.es

Departamento de Matemáticas, Universidad de Murcia, 30100 Murcia, Spain

E-mail address: jsimon@um.es 